OPEN ACCESS

Edited by:

Weiren Zhu,

Shanghai Jiao Tong University, China

Reviewed by:

Jun Ding,

East China Normal University, China Junming Zhao,

Nanjing University, China

${ }^{*}$ Correspondence:

Liansheng Wang

wlsw/s1982@126.com

Specialty section: This article was submitted to

Optics and Photonics,

a section of the journal

Frontiers in Physics

Received: 21 May 2020

Accepted: 02 July 2020

Published: 19 November 2020

Citation:

Wang L, Xia D, Fu Q, Ding X and Wang Y (2020) Graphene-Based

Tunable Wideband Metamaterial

Absorber With Polarization Insensitivity

and Wide Incident Angle.

Front. Phys. 8:303

doi: 10.3389/fphy.2020.00303

\section{Graphene-Based Tunable Wideband Metamaterial Absorber With Polarization Insensitivity and Wide Incident Angle}

\author{
Liansheng Wang ${ }^{1 *}$, Dongyan Xia ${ }^{2}$, Quanhong Fu ${ }^{3}$, Xueyong Ding ${ }^{1}$ and Yuan Wang ${ }^{1}$ \\ ${ }^{1}$ Science and Polytechnic Department, Sanya University, Sanya, China, ${ }^{2}$ Finance and Economics Department, Sanya \\ University, Sanya, China, ${ }^{3}$ Science Department, Northwestern Polytechnical University, Xi'an, China
}

A graphene-based tunable wideband metamaterial absorber with polarization insensitivity and wide incident angle is presented in this paper. The results show that the absorption is over $90 \%$ from 5 to $8 \mathrm{GHz}$ when the Fermi level of graphene is $0.5 \mathrm{eV}$, and the absorption can be tuned by electrically changing the Fermi level of graphene. The mechanism of wideband and tunable absorption is explained by calculating the normalized input impedance and monitoring the surface current. Finally, the results adequately verify that the absorption of metamaterial absorber has the advantages of polarization insensitivity and wide incident angle. Thus, it holds great potential application value in many fields such as electromagnetic stealth, electromagnetic shielding, communication, and so on.

Keywords: wideband, tunable, graphene, metamaterial absorber, wide-incident-angle

\section{INTRODUCTION}

In recent years, metamaterial, a kind of artificially structured material has attracted wide attention due to its unique electromagnetic properties [1-3]. With the development of research on metamaterial, researchers discovered that metamaterial has great potential in the fields of super lens, electromagnetic stealth, optical black hole, polarization converter, perfect electromagnetic absorption, and many more [4-8]. Among the above applications, metamaterial absorber was proposed by Landy et al. [9]. Since then, the research of metamaterial absorber has attracted extensive attention because of its perfect electromagnetic absorption property, ultrathin microstructure, and great potential in military and civil applications. Currently, the research of metamaterial absorber mainly focuses on the wide incident angle, polarization independency, tunability, and multiband absorption [10-14]. However, the wideband metamaterial absorber is more suitable for the application of practical electromagnetic environment. At present, many methods have been used to expand the absorption bandwidth of metamaterial absorber, such as vertical superposition of different resonant units, fractal structure, and magnetic media and so on [15-18]. Among them, the combination of lumped element, plasma Brewster enhancement, and strong coupling effect with metamaterial absorber is a very effective method to realize wideband metamaterial absorber [19-21]. 
Compared with the traditional passive metamaterial absorber, the active metamaterial absorber can realize the active adjustment of its electromagnetic property by changing the parameters of lumped components or the electromagnetic property of the intermediate medium, so it has more application prospects in practice.

Based on the electrically tunable conductivity of graphene, a graphene-based tunable wideband metamaterial absorber with polarization insensitivity and wide incident angle is designed in this paper. The results show that the absorption of metamaterial absorber is more than $90 \%$ from 5 to $8 \mathrm{GHz}$ when the Fermi energy level of graphene is $0.5 \mathrm{eV}$, and the absorption can be tuned by electrically changing the Fermi level of graphene. Further study shows that the absorption property of metamaterial absorber has the advantages of polarization insensitivity and wide incident angle.

\section{THE ELECTRICALLY TUNABLE CONDUCTIVITY OF GRAPHENE}

Graphene is a kind of two-dimensional planar crystal with very high electron and hole mobility. Its conductivity can be regulated by changing the external bias voltage applied on graphene, so graphene is widely used in tunable microwave and terahertz devices. When there is no bias magnetic field applied on graphene, its conductivity from microwave to visible band can be expressed by Kubo formula [22]:

$$
\begin{gathered}
\sigma\left(\omega, \mu_{c}\right)=\frac{-i e^{2}(\omega+i 2 \Gamma)}{\pi \hbar^{2}}\left\{\frac{1}{(\omega+i 2 \Gamma)^{2}} \int_{0}^{\infty} \varepsilon\left[\frac{\partial f_{d}(\varepsilon)}{\partial \varepsilon}-\frac{\partial f_{d}(-\varepsilon)}{\partial \varepsilon}\right] d \varepsilon-\right. \\
\left.\int_{0}^{\infty} \varepsilon\left[\frac{f_{d}(-\varepsilon)-f_{d}(\varepsilon)}{(\omega+i 2 \Gamma)^{2}-4(\varepsilon / \hbar)^{2}}\right] d \varepsilon\right\} \\
f_{d}(\varepsilon)=\left[\exp \left(\varepsilon-\mu_{c}\right) /\left(k_{B} T\right)+1\right]^{-1}
\end{gathered}
$$

In the formulas (1) and (2), $k_{B}$ is the Boltzmann constant, $\hbar$ is the reduced Dirac constant, $f_{d}(\varepsilon)$ is the Fermi Dirac distribution function, $\mu_{c}$ is the Fermi level of graphene, Tis the Kelvin temperature, and $\Gamma=5 \times 10^{12} \mathrm{~Hz}$ is the carrier scattering rate. According to formulas (1) and (2), the calculated conductivity of graphene with different Fermi levels is shown in Figure 1.

It can be seen from Figure 1 that the conductivity of graphene increases with the increasing of its Fermi level. The main method of changing the Fermi level of graphene is applying bias voltage on it. The Fermi level of graphene under different bias voltages applied on can be expressed as:

$$
E_{\text {bias }}=\frac{e}{\varepsilon_{0} \pi \hbar^{2} v_{F}^{2}} \int_{0}^{\infty} \varepsilon\left[f_{d}(\varepsilon)-f_{d}\left(\varepsilon+2 \mu_{c}\right)\right] d \varepsilon
$$

In formula (3), $E_{b i a s}$ is the bias voltage applied on graphene, $v_{F}=$ $10^{6} \mathrm{~m} / \mathrm{s}$ is the velocity of the electron. Graphene can be set as a material with the thickness of $0.001 \mu \mathrm{m}$, and the conductivity as shown in Figure 1.

\section{MODEL DESIGN}

The unit cell of our graphene-based tunable wideband metamaterial absorber with polarization insensitivity and wide incident angle is presented in Figure 2. The unit cell consists of five layers: fractal tree metal loaded with graphene, FR4 medium $(\varepsilon=4.3, \tan \delta=0.025)$, cross metal, FR4 medium, and metallic ground, in which the fractal tree metal and cross metal layer are connected by passing metal rods through the middle FR4 layer, as illustrated in Figure 2. The metal part of unit cell is composed of copper (). The optimized parameters of the unit cell are $a=b=9 \mathrm{~mm}, c=d=e=0.6 \mathrm{~mm}, f=8.5 \mathrm{~mm}$, $t_{1}=0.6 \mathrm{~mm}, t_{2}=2 \mathrm{~mm}, t_{3}=0.1 \mathrm{~mm}$. Doped silicon is used as the electrode to apply bias voltage on graphene.

The modeling and numerical simulation of metamaterial absorber is performed with Microwave Studio CST. At the simulation process, the boundary conditions of $x$ and $y$ directions are set as unit cell, the $z$ direction is set as open, All+Floquet ports are used to simulate the incoming and outgoing waves. The electromagnetic parameters are calculated by using frequency domain electromagnetic solver.

\section{RESULTS AND DISCUSSION}

When the incident electromagnetic waves enter into the proposed metamaterial absorber, the magnetic and electric resonances will be produced independently. The electromagnetic power of incident waves gets absorbed gradually by the dielectric loss and the loss of lumped graphene. When the absorption of metamaterial absorber reaches near perfect absorption, it obtains a state such that the permittivity equals the permeability and at the same time matches the impedance to free space. In a more direct perspective, the absorption of metamaterial absorber can be expressed as:

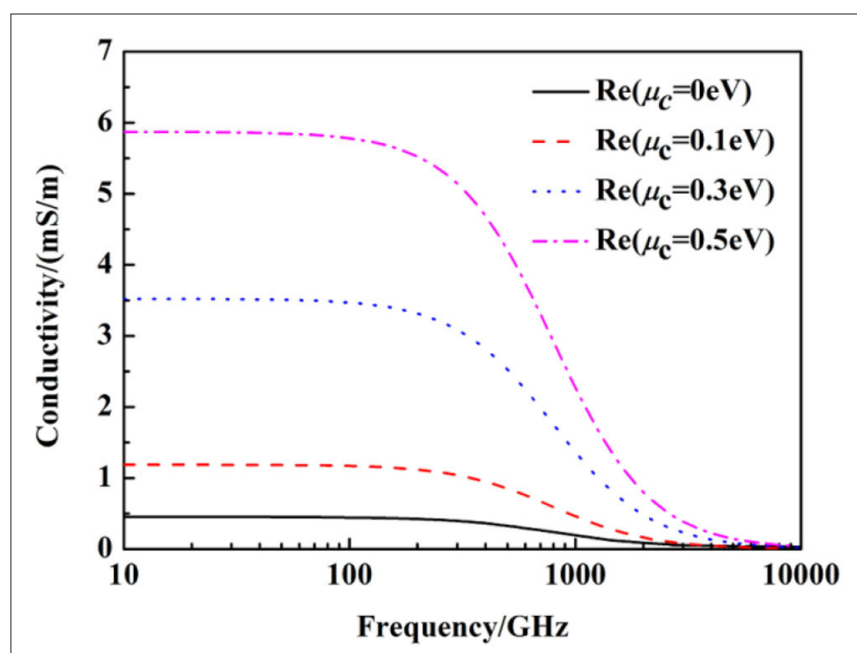

FIGURE 1 | The conductivity of graphene under different Fermi levels. 

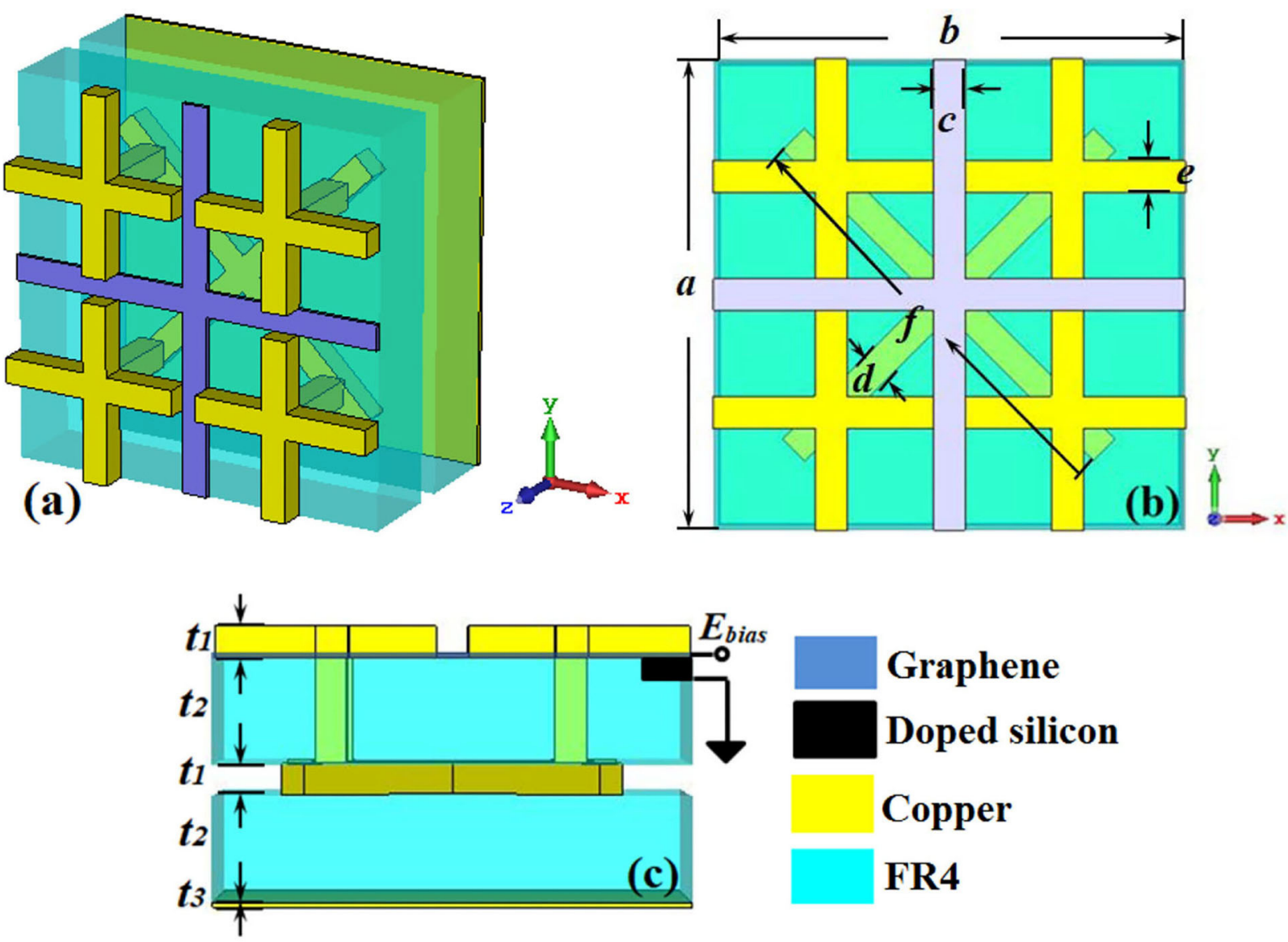

\section{Copper}

FR4

FIGURE 2 | The diagram of the metamaterial absorber unit cell: (A) perspective view, (B) front view, (C) side view.

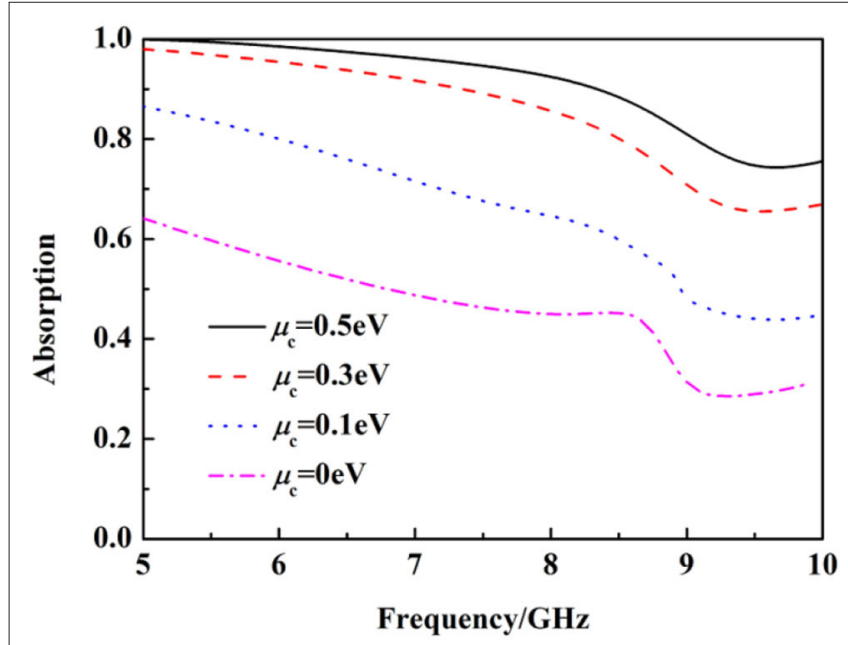

FIGURE 3 | The absorption of the metamaterial absorber under different Fermi levels.

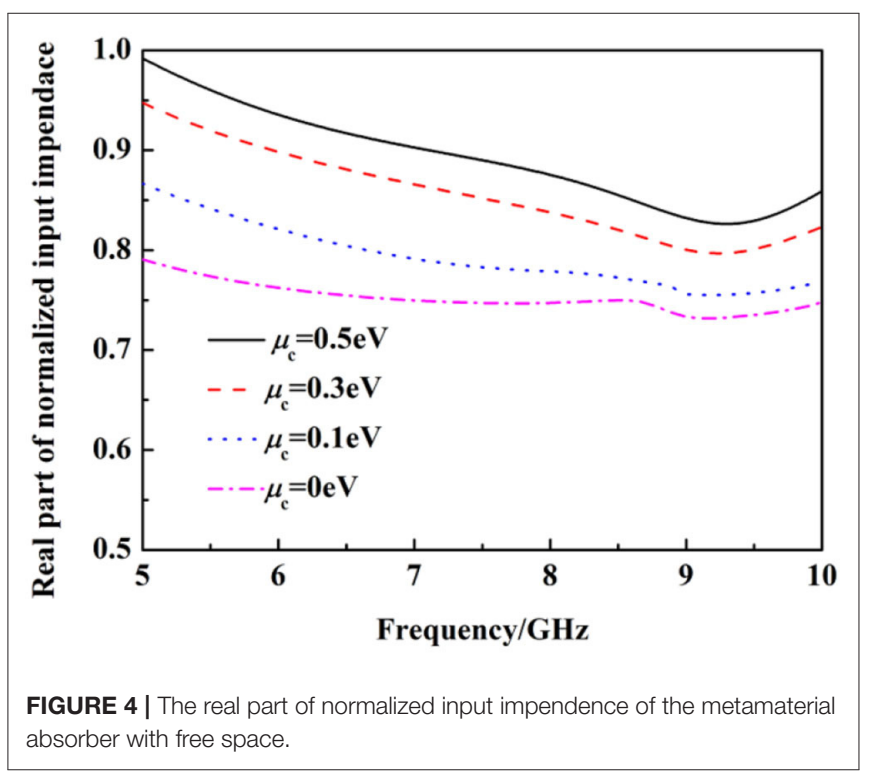



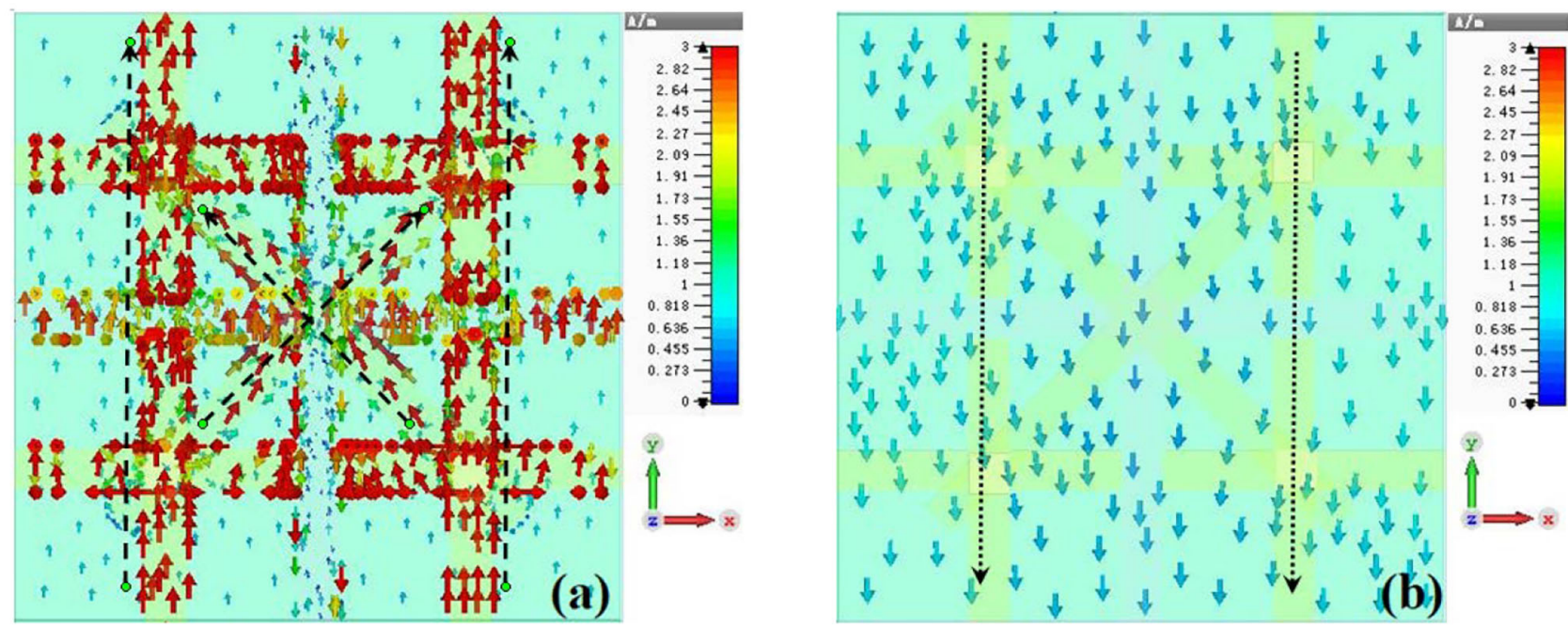

FIGURE 5 | (a,b) The surface current distribution of the metamaterial absorber at $5 \mathrm{GHz}$ with the Fermi level of graphene at 0.5 eV.
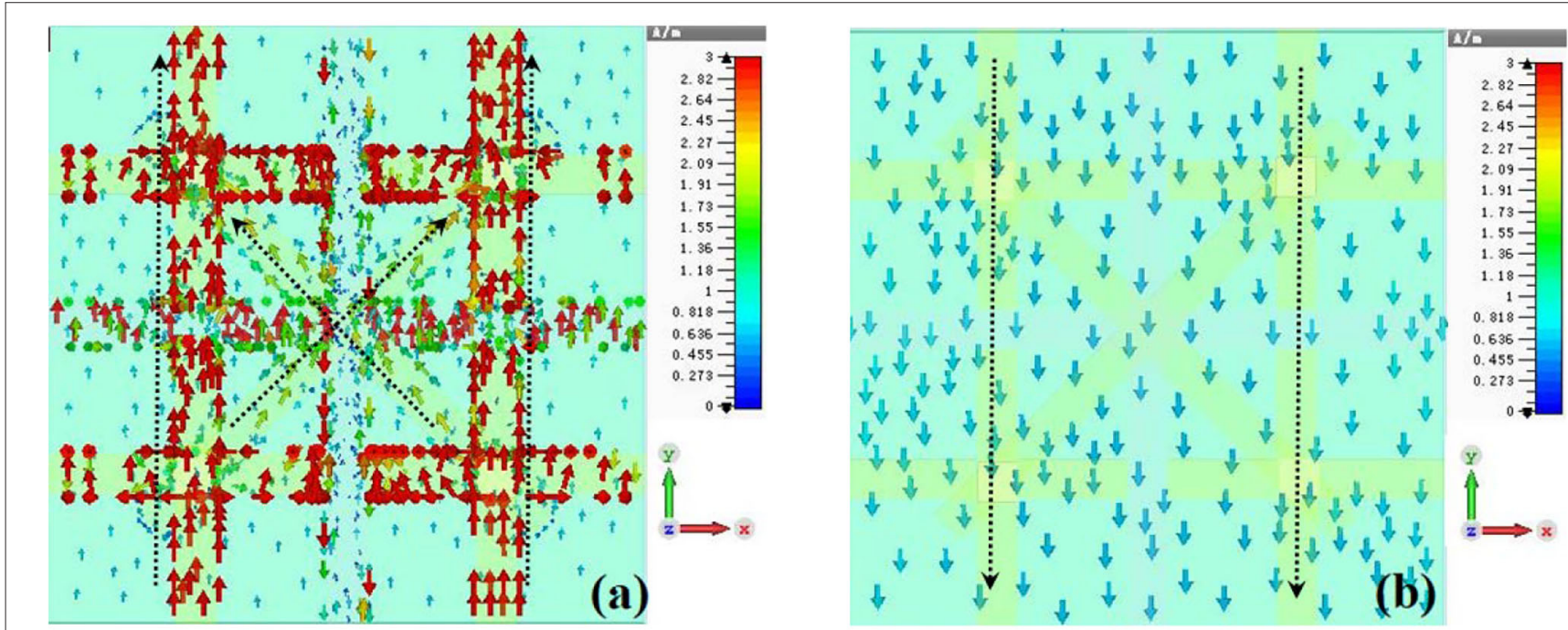

FIGURE 6 | (a,b) The surface current distribution of the metamaterial absorber at $6 \mathrm{GHz}$ with the Fermi level of graphene at 0.5 eV.

$$
A(\omega)=1-R(\omega)-T(\omega)=1-\left|S_{11}(\omega)\right|^{2}-\left|S_{21}(\omega)\right|^{2}
$$

Since the metallic ground of unit cell prevents the incident electromagnetic wave transmission, the above calculation formula of absorption can be simplified as:

$$
A(\omega)=1-R(\omega)=1-\left|S_{11}(\omega)\right|^{2}
$$

According to formula (5), we calculate the absorption of metamaterial absorber under different Fermi levels, as shown in Figure 3. It can be seen from Figure 3 that the absorption of metamaterial absorber exceeds $90 \%$ from 5 to $8 \mathrm{GHz}$ when the Fermi level of graphene is at $0.5 \mathrm{eV}$, and the absorption of metamaterial absorber from 5 to $8 \mathrm{GHz}$ gradually decreases with the decreasing of the Fermi level of graphene.

At present, the theory of equivalent medium can effectively explain the physical mechanism of metamaterial absorber [23]. According to the theory of equivalent medium, the metamaterial absorber can be regarded as an equivalent medium, which can be characterized by the equivalent permittivity $\varepsilon(\omega)$ and equivalent permeability $\mu(\omega)$. According to the calculation, formula of normalized input impedance $\mathrm{Z}(w) / \mathrm{Z}_{0}=\sqrt{u(w) / \varepsilon(w)}$, when the equivalent permittivity $\varepsilon(\omega)$ and the equivalent 
permeability $\mu(\omega)$ of metamaterial absorber are identical, the normalized input impedance of metamaterial absorber with free space equals one, and then the reflection of metamaterial absorber to the incident wave reaches zero. As an outcome, the perfect absorption of metamaterial absorber will be realized. According to the $S_{11}$ and $S_{21}$ parameters extracted by simulation, the normalized input impedance $\mathrm{Z}_{1}(\omega) \quad\left(\mathrm{Z}_{1}(\omega)=\mathrm{Z}(\omega) / \mathrm{Z}_{0}\right)$ of metamaterial absorber under different Fermi levels is calculated using the scattering parameter method [24], as shown in Figure 4. It can be seen from Figure 4 that the real part of the normalized input impedance of metamaterial absorber with free space is close to one from 5 to $8 \mathrm{GHz}$ with the Fermi energy level of graphene at $0.5 \mathrm{eV}$. This indicates that the metamaterial absorber has a good impedance matching with free space and achieves the condition of perfect absorption. At the same time, the real part of the normalized input impedance of metamaterial absorber with free space decreases gradually with the increasing of frequency, which leads to the decreasing of absorption.

In order to analyze the mechanism of wideband absorption, the surface current distribution of metamaterial absorber at 5, 6, and $7 \mathrm{GHz}$ with the Fermi level of graphene at $0.5 \mathrm{eV}$ is monitored, as shown in Figures 5, 7. It can be seen from Figures $5 \mathrm{~A}, 6 \mathrm{~A}, 7 \mathrm{~A}$ that the generated surface current at the top fractal tree metal layer and the middle cross metal layer flows upward (although the surface current of the cross metal structure at $7 \mathrm{GHz}$ is downward, it is very weak). This will lead
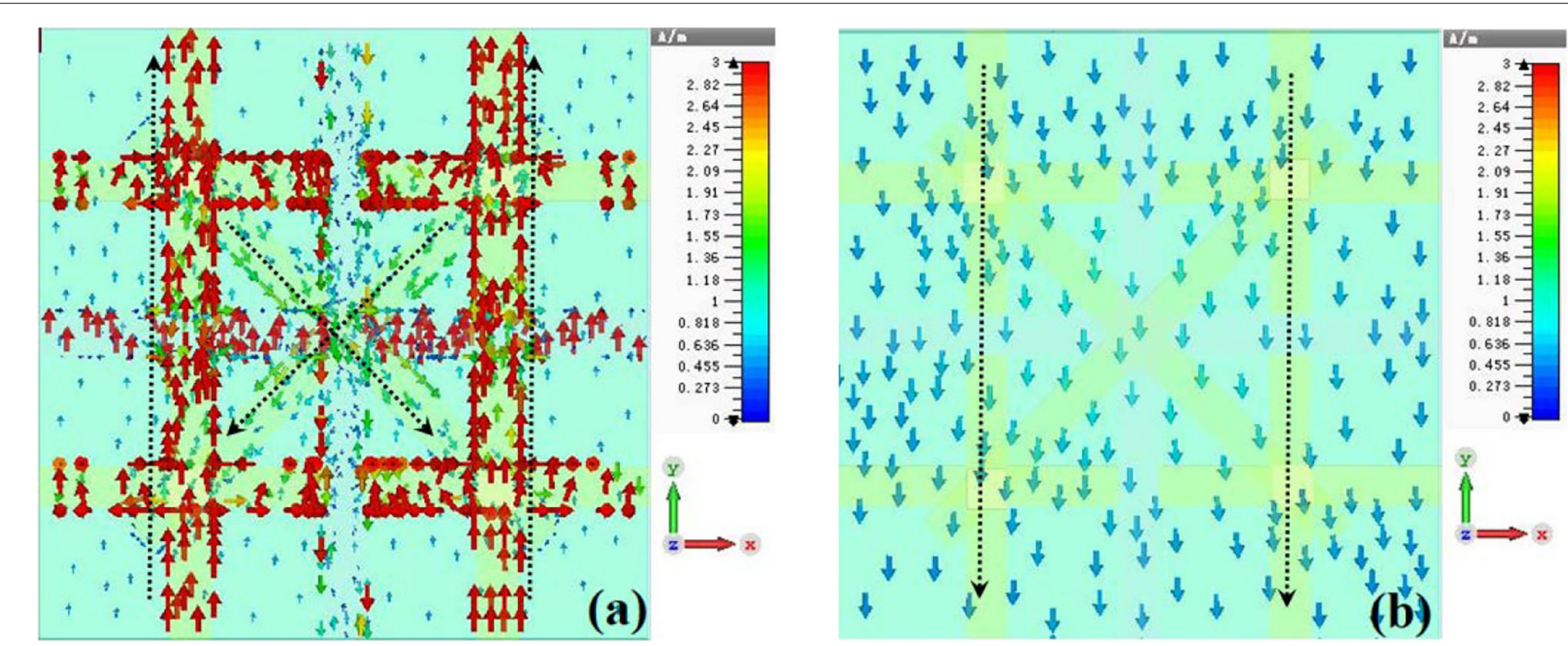

FIGURE 7 | (a,b) The surface current distribution of the metamaterial absorber at $7 \mathrm{GHz}$ with the Fermi level of graphene at 0.5 eV.
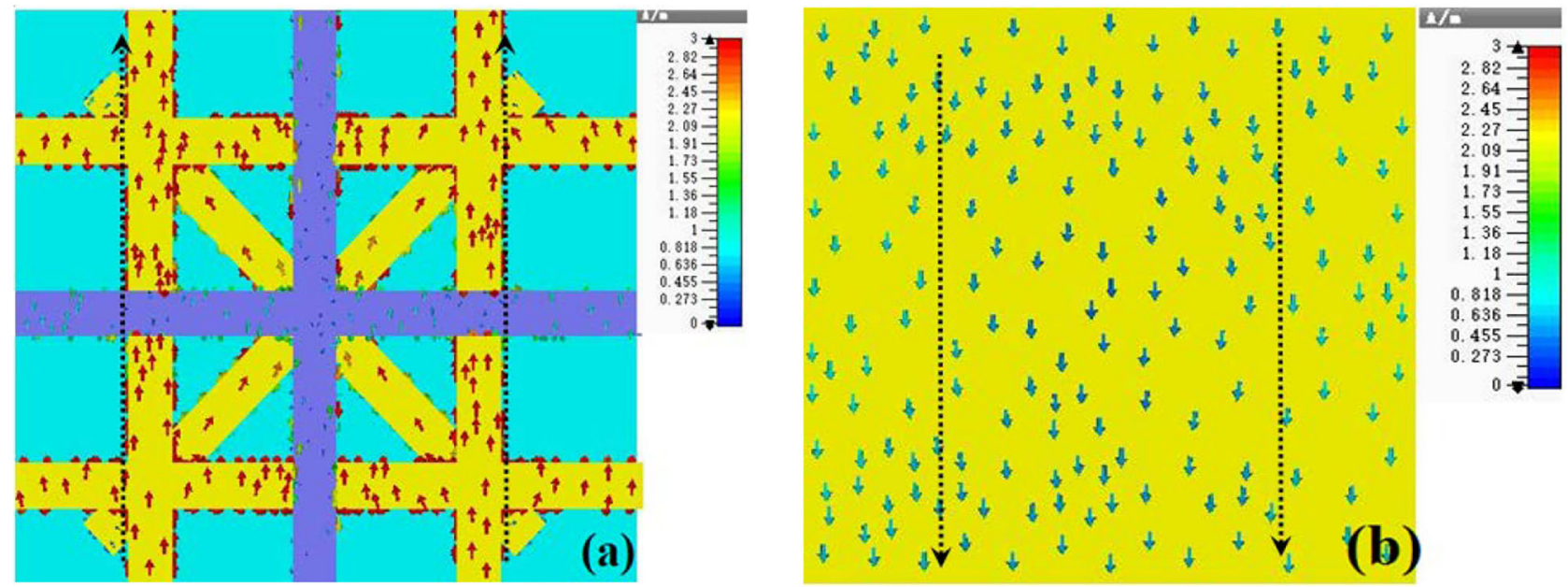

FIGURE 8 | (a,b) The surface current distribution of the metamaterial absorber at $5 \mathrm{GHz}$ with the Fermi level of graphene at 0 eV. 
to the alternating accumulation of charges in the $y$ direction, and then form electric dipole resonance [25]. It can be seen from Figures 5B, 6B, 7B that the generated surface current at the bottom metal substrate flows downward, which is opposite to the current direction of the top fractal tree metal layer and the cross metal layer. This antiparallel current forms a current circuit, which can cause a strong magnetic response resonance [26]. The magnetic resonance and electrical resonance are realized simultaneously at 5, 6, and $7 \mathrm{GHz}$ and achieve the condition of perfect absorption. The wideband absorption is caused by the overlay of different resonance frequencies.

In order to further analyze the mechanism of tunable absorption, the surface current of metamaterial absorber at 5
$\mathrm{GHz}$ with the Fermi level of graphene at $0 \mathrm{eV}$ is monitored, as shown in Figure 8. It can be seen from Figure 8 that the metamaterial absorber generates electric resonance and magnetic resonance under the action of incident microwave $[25,26]$. However, the excited surface currents on the fractal tree metal layer and the cross metal layer are weaker than the one at Fermi level of $0.5 \mathrm{eV}$ (Figure 5), so the generated electromagnetic resonance is very weak, this results in lower absorption.

The absorption of metamaterial absorber under different polarization angles with the Fermi level of graphene at $0.5 \mathrm{eV}$ is calculated, as shown in Figure 9. It can be seen from Figure 9 that the absorption of the metamaterial absorber under different

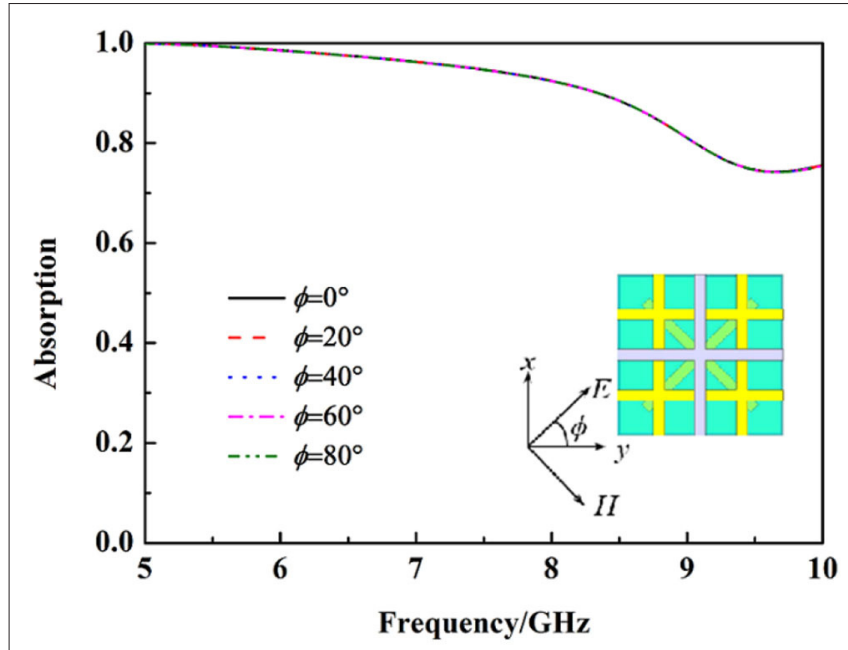

FIGURE 9 | The absorption of the metamaterial absorber under different polarization states with the Fermi level of graphene at $0.5 \mathrm{eV}$.

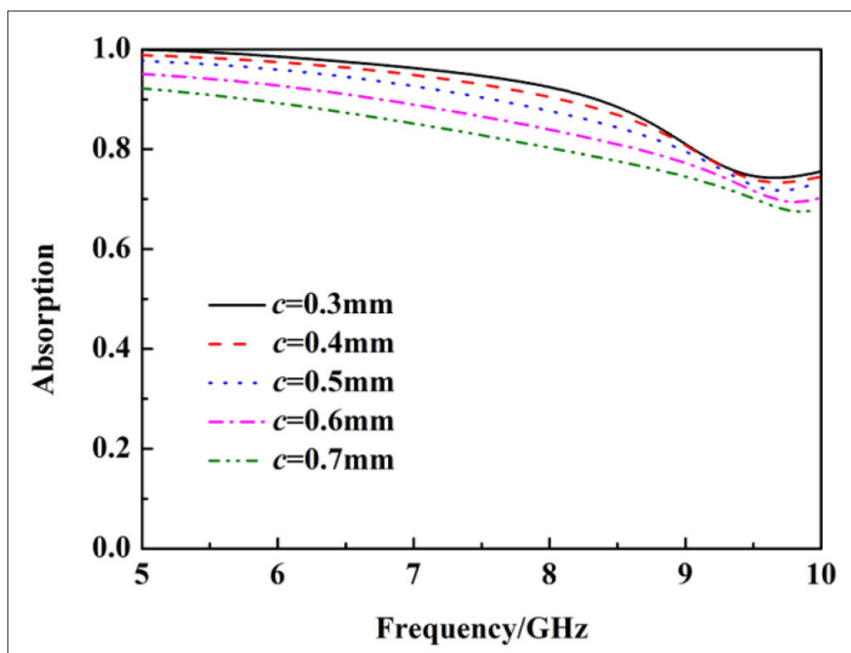

FIGURE 11 | The absorption of the metamaterial absorber under different structural dimension parameters $c$ with the Fermi level of graphene at $0.5 \mathrm{eV}$.
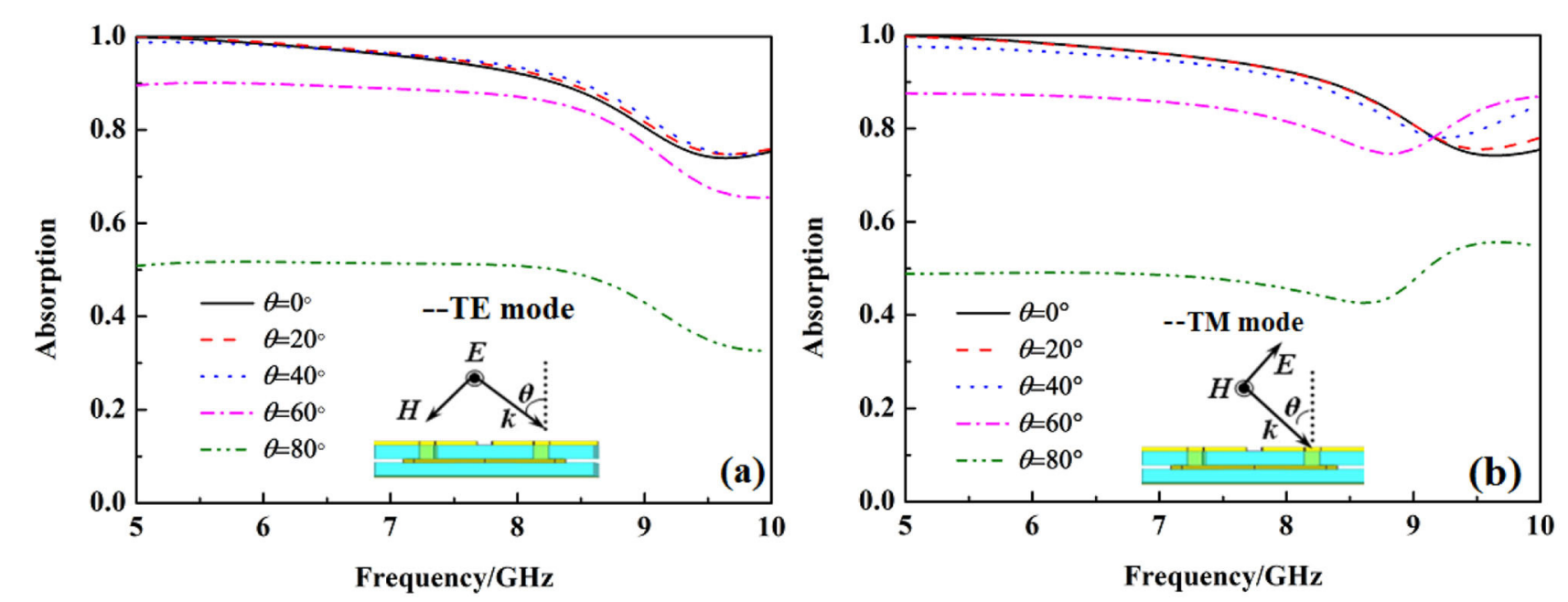

FIGURE 10 | The absorption of the metamaterial absorber under different incident angles with the Fermi level of graphene at 0.5 eV: (a) transverse electric (TE) mode, (b) transverse magnetic (TM) mode. 


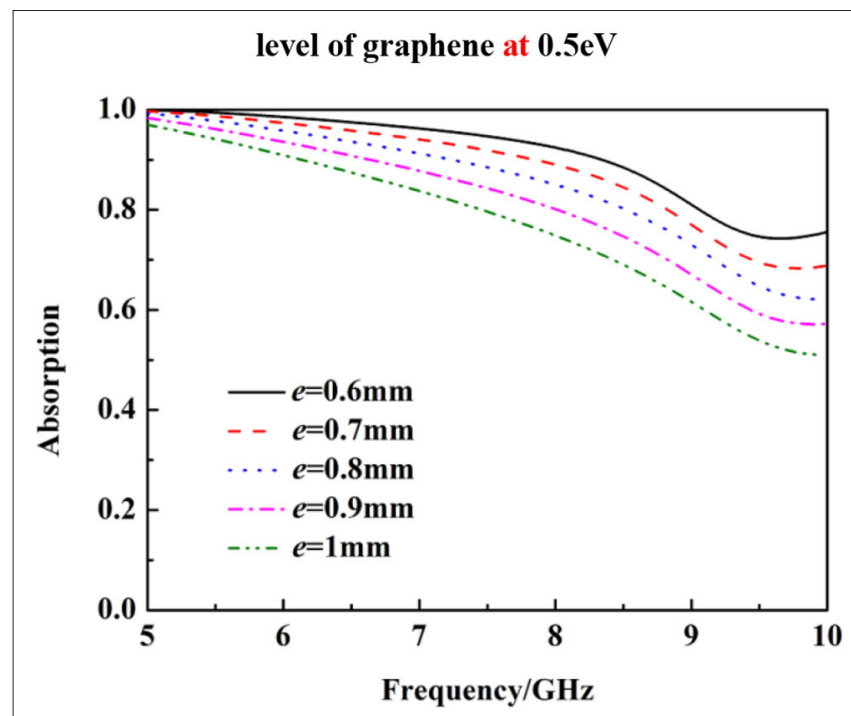

FIGURE 12 | The absorption of the metamaterial absorber under different structural dimension parameters e with the Fermi level of graphene at $0.5 \mathrm{eV}$.

polarization angles is the same due to the rotational symmetry of the unit cell.

The absorption of the metamaterial absorber under different incident angles with the Fermi level of graphene at $0.5 \mathrm{eV}$ is calculated, as shown in Figure 10. It can be seen from Figure 10 that the absorption of the metamaterial absorber from 5 to $8 \mathrm{GHz}$ gradually decreases with the incident angle increasing from 0 to $60^{\circ}$ at transverse electric (TE) mode and transverse magnetic (TM) mode, but it can still be up to $90 \%$ from 5 to 8 $\mathrm{GHz}$ when the incident angle is $60^{\circ}$. The results show that the absorption of metamaterial absorber has the advantage of wide incident angle.

\section{REFERENCES}

1. Cui TJ, Smith DR. Metamaterials: Theory, Design, Applications. New York, NY: Springer (2010).

2. Alastair P, Hibbins BR, Evans J, Sambles R. Experimental verification of designer surface. Plasmon Sci. (2005) 308:670-2. doi: 10.1126/science.1109043

3. Uchida K, Adachi H, Kikuchi D, Ito S, Qiu Z, Maekawa S, et al. Generation of spin currents by surface plasmon resonance. Nat Commun. (2015) 6:5910. doi: 10.1038/ncomms6910

4. Quader S, Zhang J, Akram MR, Zhu W. Graphene-based highefficiency broadband tunable linear-to-circular polarization converter for terahertz waves. IEEE J Select Topics Quant Electron. (2020) 26:4501008. doi: 10.1109/JSTQE.2020.2969566

5. Zhang J, Wei X, Rukhlenko ID, Chen HT, Zhu W. Electrically tunable metasurface with independent frequency and amplitude modulations. ACS Photonics. (2020) 7:265-71. doi: 10.1021/acsphotonics.9b 01532

6. Zhang J, Wei X, Premaratne M, Zhu W. Experimental demonstration of electrically tunable broadband coherent perfect absorber based on graphene-electrolyte-graphene sandwich structure. Photonics Res. (2019) 7:868-74. doi: 10.1364/PRJ.7.000868

7. Liu B, Zhu W, Gunapala SD, Stockman MI, Premaratne M. Open resonator electric spaser. ACS Nano. (2017) 11:12573-82. doi: 10.1021/acsnano.7b06735
The structural size of the metamaterial absorber has an important influence on its absorption. The absorption of the metamaterial absorber under different graphene width $c$ and fractal tree metal linewidth $e$ with the Fermi level of graphene at $0.5 \mathrm{eV}$ is calculated, as shown in Figures 11, 12. It can be seen from Figures 11, 12 that the absorption of the metamaterial absorber gradually decreases with the increasing of structure size parameters $c$ and $e$.

\section{SUMMARY}

To summarize, a graphene-based tunable wideband metamaterial absorber with polarization insensitivity and wide incident angle is presented in this paper. The metamaterial absorber provides $>90 \%$ absorption from 5 to $8 \mathrm{GHz}$ when the Fermi level of graphene is $0.5 \mathrm{eV}$. The absorption of the metamaterial absorber can be tuned electrically by applying an external bias voltage on graphene. Simulation results unveil that the response of the absorber presents a remarkably high absorption for a wide range of incident angles and is insensitive to polarization angle but has a relationship with its structure size parameters. The metamaterial absorber delivers the advantages of wideband, tunable, polarization insensitivity, and wide incident angle. It has great potential application value in the fields of electromagnetic stealth, electromagnetic shielding, communication, and so on.

\section{DATA AVAILABILITY STATEMENT}

The raw data supporting the conclusions of this article will be made available by the authors, without undue reservation.

\section{AUTHOR CONTRIBUTIONS}

All authors listed have made a substantial, direct and intellectual contribution to the work, and approved it for publication.

8. Akram MR, Ding G, Chen K, Feng Y, Zhu W. Ultra-thin single layer metasurfaces with ultra-wideband operation for both transmission and reflection. Adv Mater. (2020) 32:1907308. doi: 10.1002/adma. 201907308

9. Landy NI, Sajuyigbe SJ, Mock J, Smith DR, Padilla WJ. Perfect metamaterial absorber. Phys Rev Lett. (2008) 100:207402. doi: 10.1103/PhysRevLett.100.207402

10. Aydin K, Ferry VE, Briggs RM, Atwater HA. Broadband polarizationindependent resonant light absorption using ultrathin plasmonic super absorbers. Nat Commun. (2011) 2:517. doi: 10.1038/ ncomms 1528

11. Xu HX, Wang GM, Qi MQ, Liang JG, Gong JQ, Xu ZM. Triple-band polarization insensitive wide-angle ultra-miniature metamaterial transmission line absorber. Phys Rev B. (2012) 86:205104. doi: 10.1103/PhysRevB.86.205104

12. Ding F, Cui YX, Ge XC, Jin Y, He SL. Ultra-broadband microwave metamaterial absorber. Appl Phys Lett. (2012) 100:103506. doi: 10.1063/1.3692178

13. Argyropoulos C, Le KQ, Mattiucci N. Broadband absorbers and selective emitters based on plasmonic Brewster metasurfaces. Phys Rev B. (2013) 87:205112. doi: 10.1103/PhysRevB.87.205112

14. Li L, Yang Y, Liang C. Ultrathin multiband gigahertz metamaterial absorbers. J Appl Phys. (2011) 110:063702. doi: 10.1063/1.3608246 
15. Othman MAK, Guclu C, Capolino F. Graphene-based tunable hyperbolic metamaterials and enhanced near-field absorption. Opt Express. (2013) 21:7614-32. doi: 10.1364/OE.21.007614

16. Cao T, Wei C, Simpson RE, Zhang L, Cryan MJ. Rapid phase transition of a phase-change metamaterial perfect absorber. Opt Mater Express. (2013) 3:1101-10. doi: 10.1364/OME.3.001101

17. Kebin F, Strikwerda AC, Zhang X, Averitt RD. Three-dimensional broadband tunable terahertz metamaterials. Phys Rev B. (2013) 87:161104. doi: 10.1103/PhysRevB.87.161104

18. Hedayati MK, Zillohu AU, Strunskus T, Faupel F, Elbahri M. Plasmonic tunable metamaterial absorber as ultraviolet protection film. Appl Phys Lett. (2014) 104:041103. doi: 10.1063/1.4863202

19. Huang Y, Wen G, Zhu W, Li J, Si LM, Premaratne M. Experimental demonstration of a magnetically tunable ferrite based metamaterial absorber. Opt Express. (2014) 22:16408-17. doi: 10.1364/OE.22.016408

20. Wang P, Casadei F, Shan S, Weaver JC, Bertoldi K. Harnessing buckling to design tunable locally resonant acoustic metamaterials. Phys Rev Lett. (2014) 113:014301. doi: 10.1103/PhysRevLett.113.014301

21. Tuong PV, Park JW, Rhee JY, Kim KW, Jang WH, Cheong H, et al. Polarization-insensitive and polarization-controlled dual-band absorption in metamaterials. Appl Phys Lett. (2013) 102:081122. doi: 10.1063/1.47 94173

22. Fallahi A, Perruisseau-Carrier J. Design of tunable biperiodic graphene metasurfaces. Phys Rev B. (2012) 86:195408. doi: 10.1103/PhysRevB.86.195408
23. Chen HT. Interference theory of metamaterial perfect absorbers. Opt Express. (2012) 20:7165-72. doi: 10.1364/OE.20.007165

24. Smith DR, SchultzS. Determination of negative permittivity and permeability of metamaterials from reflection and transmission coefficients. Phys Rev B. (2002) 65:195104. doi: 10.1103/PhysRevB.65.195104

25. Chen JF, Huang XT, Zerihun G. Polarization-independent, thin, broadband metamaterial absorber using double-circle rings loaded with lumped resistances. J Electron Mater. (2015) 44:4269-74. doi: 10.1007/s11664-015-3951-x

26. Bhattacharyya S, Ghosh S, Srivastava KV. Triple band polarizationindependent metamaterial absorber with bandwidth enhancement at X-band. J Appl Phys. (2013) 114:094514. doi: 10.1063/1.4820569

Conflict of Interest: The authors declare that the research was conducted in the absence of any commercial or financial relationships that could be construed as a potential conflict of interest.

Copyright (c) 2020 Wang, Xia, Fu, Ding and Wang. This is an open-access article distributed under the terms of the Creative Commons Attribution License (CC BY). The use, distribution or reproduction in other forums is permitted, provided the original author(s) and the copyright owner(s) are credited and that the original publication in this journal is cited, in accordance with accepted academic practice. No use, distribution or reproduction is permitted which does not comply with these terms. 